\title{
Encyclopedia
}

\section{Decentralized Energy Production in Urban Areas}

Created by: Dhruv Suri

Revised by: Catherine Yang

Version received: 24 October 2019

check for

updates

This article discusses the applicability of Ducted Wind Turbines for decentralized energy generation in an urban environment. Ducted Wind Turbines overcome various constraints posed by urban infrastructure including reduced wind speed, the effect of the atmospheric boundary layer as well as lesser space availability. Ducted Wind Turbines presents a new way of sourcing energy at a local level, aiding community-based models of energy distribution and consumption. The main aim of the present letter is to propose Ducted Wind Turbines as a precursor for energy production in urban, peri-urban as well as rural areas around the world.

\section{Introduction}

Rising incomes and an additional 1.7 billion people mostly added to urban areas in developing countries will push up global energy demand by $25 \%$ in the period to 2040 [1]. The convergence of affordable and reliable renewable energy technologies with effective policy implementation at the international and national levels is set to push societies closer towards meeting many of the world's sustainable development goals. While cost-effective renewable energy technologies enable community-based models of energy production, shifting supply and demand calls for continuous improvement in energy efficiency across both private and public sectors. In order for wind technology to be a viable contender, nextgeneration wind turbines should increase both performance and reliability, thus lowering the levelized cost of electricity (LCOE). Wind power accounted for $4.2 \%$ of the total energy generated in 2017 and is expected to account for a quarter of all energy generation by 2040 .

The share of wind energy in global energy supply underscores the need for affordable, reliable and scalable wind energy systems. A vast majority of wind energy production today is from offshore and onshore wind turbines located far away from urban areas which account for $70 \%$ of the global electricity demand [2]. As the demand for energy from urban areas increases, the interplay between the production and consumption of electricity has a key role to play in the reliability of wind energy systems. To account for this increase in electricity demand, the prospect of urban energy production has been explored $[3,4,5,6]$. Energy production in urban areas will help reduce transmission losses and significantly lower the cost of distribution infrastructure [7]. Moreover, localized energy production will also help increase the global energy intensity, a key aspect towards attaining the Sustainable Development Goals. The integration of wind energy production with the urban landscape presents a number of challenges. Firstly, the mean velocity of wind currents is reduced due to surface roughness, topography, and proximity to buildings and trees [8]. The increase in turbulence also causes rapid fluctuations in the direction of the wind [9]. Secondly, the limited availability of space imposes a constraint on the size of the wind turbine. Lastly, an urban environment necessitates the use of simple and ergonomic design; large wind turbines have complex yaw drive mechanisms to align the rotor to the wind and thus such systems cannot be integrated into an urban landscape. The need for an efficient, compact and decentralized wind energy system has put Ducted Wind Turbines (DWTs) at the forefront of innovation efforts. A schematic of a DWT and horizontal axis wind turbine (HAWT) is shown in Fig. 1. 

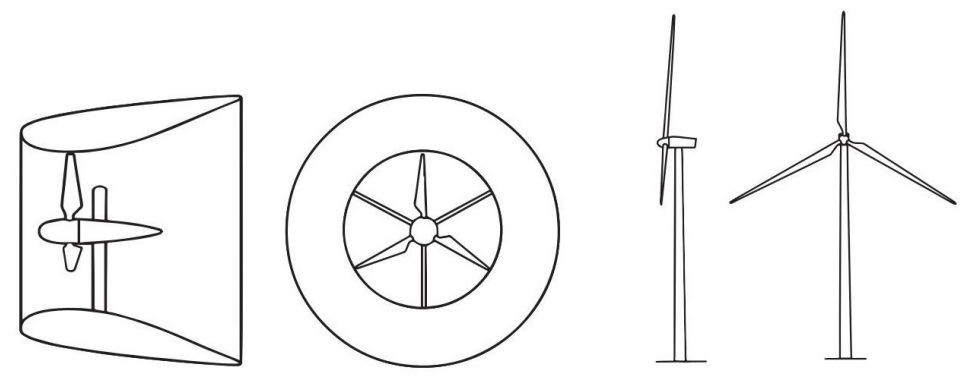

Figure 1: Schematic of a DWT (left) and HAWT (right) [10]

The first theoretical analysis of DWTs can be found in the pioneering work of Lilley and Rainbird in 1956 [11]. Subsequently, a number of researchers carried out experimental studies on the augmentation of DWTs in the seventies; Foreman et al [12] investigated the use of boundary layer controlled diffusion for enhancing the augmentation imparted by a duct. The formative work of Igra [13] concluded that the performance of a DWT was far superior as compared to a bare wind turbine of the same diameter. He also developed a one-dimensional analytical model which constituted the basis for many future works. Van Bussel [14] found that the power extracted by a DWT can exceed the Betz limit by a factor of 2.5. Aranake and Duraisamy [15] analyzed the performance of DWT at low wind speeds and found that the augmentation imparted by the duct on the flow aids the DWT in harnessing electricity even in low wind speed environments. Creswell et al [16] investigated the effect of flow yaw on the power coefficient of the DWT. The study concluded that DWT show increased performance even when the incoming flow is not aligned perpendicular to the rotor plane. This characteristic property of DWT enables them to be used in urban environments where the direction of the wind does not remain constant for prolonged periods. In addition to the aforementioned literature on DWT, a number of studies were conducted based on analytical, numerical and experimental methods over the course of almost forty years

$[17,18,19,20,21,22,23]$. These studies show that a greater amount of power can be generated by using DWTS as opposed to bare rotors. Moreover, aeroacoustic analyses conducted by several researchers $[24,25]$ determined that the quiet operation of ducted systems as opposed to bare rotors makes it a viable technology for energy production in urban environments.

\section{Discussion}

The interdependence of distribution and consumption highlights yet another key aspect in the growing energy network of the world: electricity access. The engineering and economic aspect of energy distribution in developing countries have been addressed by a number of high-quality studies. While both techno-economic, as well as socio-political aspects of mini-grids and electricity access, have been discussed, there is no specific literature paving the way for DWT to be considered as a potential technology for decentralized energy production.

As analyzed by a number of researchers, the constraints posed by the urban environment are easily overcome by DWTs; reduced wind speed due to the influence of the atmospheric boundary layer is recoupled by an increased mass flow rate through the rotor plane. Due to this increased mass flow rate, DWT can produce more power per unit area of rotor leading to lesser space utilization as compared to conventional HAWTS for the same output power and lastly, the performance of a DWT is not impacted by turbulent fluctuations leading to changes in wind direction or yaw angle. DWTs have the potential to reduce the power acquired from centralized power generators and pave the way towards sustainable cities. By reducing the dependence of urban households and industries on centralized power grids, DWTs present a new way of sourcing energy at a local level, aiding community-based models of energy distribution and consumption.

\section{References}

1. International Energy Agency. World Energy Outlook 2018; World Energy Outlook: France, 2019; pp. 1. 


\section{Keywords}

ducted wind turbines; diffuser; shroud; urban wind energy

(c) () () 2019 by the author(s). Distribute under a Creative Commans CC BY license 